\title{
Pre-solar grains from supernovae and novae
}

\author{
Sachiko Amari ${ }^{1}$ and Katharina Lodders ${ }^{2}$ \\ ${ }^{1}$ Laboratory for Space Sciences \& the Physics Department and ${ }^{2}$ Department of Earth and \\ Planetary Sciences, Washington University, St. Louis, MO 63130, USA \\ email: sa@wuphys.wustl.edu, lodders@wustl.edu
}

\begin{abstract}
Pre-solar grains from supernova ejecta - silicon carbide of type $\mathrm{X}, \mathrm{Si}_{3} \mathrm{~N}_{4}$ and lowdensity graphite - are characterized by $\mathrm{Si}$ isotopic anomalies (mainly ${ }^{28} \mathrm{Si}$ excesses), low ${ }^{14} \mathrm{~N} /{ }^{15} \mathrm{~N}$, high ${ }^{26} \mathrm{Al} /{ }^{27} \mathrm{Al}$ ratios, and occasionally by excesses in ${ }^{44} \mathrm{Ca}$ (from ${ }^{44} \mathrm{Ti}$ decay). Overall isotopic features of these $\mathrm{SiC}$ and graphite grains can be explained by mixing of inner Si-rich zones and the outer $\mathrm{C}$-and He-rich zones, but supernova models require fine tuning to account for ${ }^{14} \mathrm{~N} /{ }^{15} \mathrm{~N}$ and ${ }^{29} \mathrm{Si} /{ }^{28} \mathrm{Si}$ ratios of the grains. Isotopic ratios of $\mathrm{Zr}, \mathrm{Mo}$ and $\mathrm{Ba}$ in $\mathrm{SiC} \mathrm{X}$ grains may be explained by a neutron burst model. Some of the pre-solar nanodiamonds require a supernova origin to explain measured xenon isotopic ratios. Only a few nova grain candidates, with low ${ }^{12} \mathrm{C} /{ }^{13} \mathrm{C},{ }^{14} \mathrm{~N} /{ }^{15} \mathrm{~N}$, and high ${ }^{26} \mathrm{Al} /{ }^{27} \mathrm{Al}$ ratios, have been identified.
\end{abstract}

Keywords. meteors, meteoroids, stars: supernovae: general, stars: novae, cataclysmic variables

\section{Introduction}

Since the recovery of the first pre-solar grains in 1987 a considerable amount of data has been accumulated in this new field of astronomy. Pre-solar minerals currently known include nanodiamond (Lewis et al. 1987), SiC (Bernatowicz et al. 1987; Tang \& Anders 1988), graphite (Amari et al. 1990), Al-bearing oxides (Hutcheon et al. 1994; Nittler et al. 1994), $\mathrm{Si}_{3} \mathrm{~N}_{4}$ (Nittler et al. 1995), silicates (Messenger et al. 2003; Nguyen \& Zinner 2004; Nagashima, Krot \& Yurimoto 2004), and refractory inclusions inside graphite (Bernatowicz et al. 1991) and SiC grains (Bernatowicz, Amari \& Lewis 1992). Isotopic analyses on individual grains by secondary ion mass spectrometry indicate that a small fraction of pre-solar grains originated in supernovae (e.g., Amari \& Zinner 1997). There is a handful of grains that show characteristics of novae (Amari et al. 2001; Nittler \& Hoppe 2005). This paper summarizes information on pre-solar grains of supernova and of probable nova origins. More information can be found in several review papers (Zinner 1998; Clayton \& Nittler 2004; Lodders \& Amari 2005) and references therein.

\section{Pre-solar grains from type II supernovae}

\subsection{Oxides, SiC type $\mathrm{X}, \mathrm{Si}_{3} \mathrm{~N}_{4}$ and low-density graphite}

The major dust forming elements (e.g., Al, Ca, Mg, Si) are mainly produced and ejected by core-collapse type II supernovae ( $\mathrm{SNe}$ ) and if they condense from supernova (SN) ejecta, there should be abundant grains from SNe among pre-solar grains. However, only a few oxide grains and one olivine aggregate of probable SN origin have been identified (Choi et al. 1998; Nittler et al. 1998; Messenger, Keller \& Lauretta 2005). They show either ${ }^{16} \mathrm{O}$ or ${ }^{18} \mathrm{O}$ excesses (here and elsewhere, an excess is relative to 'normal' isotopic composition). It is odd that $\mathrm{SN}$ oxide grains are so rare because ${ }^{16} \mathrm{O}$ is the third most abundant isotope ejected from $\mathrm{SNe}$ and overall $\mathrm{SN}$ ejecta have $\mathrm{C} / \mathrm{O}<1$, so there should be many more SN oxide grains. One explanation could be that SN oxide grains are 
too small $(\ll 0.1 \mu \mathrm{m})$ to survive for long in the ISM. Such small oxide grains are also unrecoverable from meteorites during chemical pre-solar grain separation procedures.

Strangely, most of the known pre-solar grains from SNe are dominantly carbonaceous: the type X SiC (Amari et al. 1992; Hoppe et al. 2000) and $\mathrm{Si}_{3} \mathrm{~N}_{4}$ (Nittler et al. 1995), and the low-density graphite (Amari, Zinner \& Lewis 1995; Travaglio et al. 1999) grains. However, only about $1 \%$ of carbonaceous pre-solar grains originated from SNe.

The carbonaceous SN grains are characterized by ${ }^{28} \mathrm{Si}$ excesses (but a few graphite grains show ${ }^{29} \mathrm{Si}$ and/or ${ }^{30} \mathrm{Si}$ excesses), lower ${ }^{14} \mathrm{~N} /{ }^{15} \mathrm{~N}$ ratios than that of air $(272)$, and typically isotopically light $\mathrm{C}$, although their ${ }^{12} \mathrm{C} /{ }^{13} \mathrm{C}$ ratios range from 3.4 to 7200 (solar: 89). Many graphite grains show ${ }^{18} \mathrm{O}$ excesses $\left({ }^{18} \mathrm{O} /{ }^{16} \mathrm{O}\right.$ up to $185 \times$ solar $)$. Type II supernovae are the main producers of ${ }^{28} \mathrm{Si}$ so excesses of this isotope indicate a SN origin of these grains. Definite proof of their $\mathrm{SN}$ origin are excesses in ${ }^{44} \mathrm{Ca}$ from decay of ${ }^{44} \mathrm{Ti}\left(\mathrm{T}_{1 / 2}=60 \mathrm{a}\right)$ which must have been incorporated when the SiC type X and lowdensity graphite grains formed (Hoppe et al. 1996; Nittler et al. 1996). Since ${ }^{44} \mathrm{Ti}$ is produced only in explosive nucleosynthesis, the initial presence of ${ }^{44} \mathrm{Ti}$ in the grains proves supernova origin of these grains. Supernova grains also once contained radioactive ${ }^{26} \mathrm{Al}$ and ${ }^{41} \mathrm{Ca}$, which are traceable by excesses in their decay products ${ }^{26} \mathrm{Mg}$ and ${ }^{41} \mathrm{~K}$. The initial ${ }^{26} \mathrm{Al} /{ }^{27} \mathrm{Al}$ ratios range up to 0.6 in $\mathrm{SN}$ grains.

The measured isotopic compositions of the $\mathrm{SiC}$ and graphite grains require mixing of different compositional SN zones. For example, high ${ }^{26} \mathrm{Al} /{ }^{27} \mathrm{Al}$ ratios require contributions from the He-N zone (where the CNO cycle takes place), and high ${ }^{18} \mathrm{O} /{ }^{16} \mathrm{O}$ ratios in low-density graphite grains need contributions from the $\mathrm{He}-\mathrm{C}$ zone (where the triple $\alpha$ reaction operates). The $\mathrm{He}-\mathrm{N}$ and $\mathrm{He}-\mathrm{C}$ zones have to be mixed to account for the observed range of ${ }^{12} \mathrm{C} /{ }^{13} \mathrm{C}$ ratios, and the innermost Si-rich zones must contribute e.g., ${ }^{28} \mathrm{Si}$, and ${ }^{44} \mathrm{Ti}$. Mixing must also achieve $\mathrm{C} / \mathrm{O}>1$ for graphite and $\mathrm{SiC}$ condensation. Mixing models by Travaglio et al. (1999), using the SN compositions by Woosley \& Weaver (1995), reproduce the observed ${ }^{12} \mathrm{C} /{ }^{13} \mathrm{C},{ }^{18} \mathrm{O} /{ }^{16} \mathrm{O}$, and ${ }^{30} \mathrm{Si} /{ }^{28} \mathrm{Si}$ ratios, and the inferred ${ }^{26} \mathrm{Al} /{ }^{27} \mathrm{Al},{ }^{41} \mathrm{Ca} /{ }^{40} \mathrm{Ca}$, and ${ }^{44} \mathrm{Ti} /{ }^{48} \mathrm{Ti}$ ratios if jets of material from the inner Si-rich zone penetrate the intermediate O-rich zones and mix with matter of the outer C-rich zones. However, the models give less ${ }^{15} \mathrm{~N}$ and ${ }^{29} \mathrm{Si}$ than seen in the grains (Nittler et al. 1995; Travaglio et al. 1999; Hoppe et al. 2000), and improvements in stellar structure models and reassessments of reaction rates are necessary. For example, the ${ }^{15} \mathrm{~N}$ yield significantly increases when stellar rotation is taken into account, and the ${ }^{26} \mathrm{Mg}(\alpha, \mathrm{n}){ }^{29} \mathrm{Si}$ reaction, which is highly temperature dependent, is poorly determined for $T=(1-4) \times 10^{9} \mathrm{~K}$ (e.g., Travaglio et al. 1998).

The Zr, Mo, and Ba isotopes in SiC X grains were analyzed with resonant ionization mass spectrometry (Pellin et al. 1999; 2000; 2001). Four out of 6 grains have relative ${ }^{95}$ Mo and ${ }^{97}$ Mo excesses up to $1.8 \times$ solar. Such enrichments are not expected from either the $s$-process (which would enrich mass 96 and 98) nor the $r$-process (which would give the largest excess in mass 100). The excesses in ${ }^{95}$ Mo and ${ }^{97}$ Mo can be explained by a neutron burst - akin to a mini $r$-process - occurring in the He-rich zone of an exploding massive star (Meyer, Clayton \& The 2000). Excesses in ${ }^{96} \mathrm{Zr}$ and ${ }^{138} \mathrm{Ba}$ in $\mathrm{X}$ grains can also be accounted for by this process.

\subsection{Diamond}

Diamond is the most abundant carbonaceous pre-solar mineral, but its origin(s) remain enigmatic. Diamonds are only measurable as aggregates of many nano-size particles that probably came from several different types of sources, including supernovae, as seen from the Xe isotopes. The anomalous Xe (Xe-HL) in diamonds is enriched in both Light (124 and 126) and Heavy (134 and 136) isotopes (Lewis et al. 1987). These are $p$ - and $r$ - process 
only isotopes, respectively, believed to come from supernovae. It remains a puzzle why the excesses in the light and the heavy isotopes are always correlated because the $p$ and $r$-process are assumed to occur in different places within supernovae. Other heavy elements, e.g., Te and $\mathrm{Pd}$ also point to a supernova origin of diamonds (Richter, Ott \& Begemann 1998; Maas et al. 2001). The SN origin of some of the pre-solar diamonds seems to be supported by the mid-IR spectra of SN 1987A, which show a broad feature at 3.40 and $3.53 \mu \mathrm{m}$ (Meikle et al. 1989), consistent with the identification of surface hydrogenated diamond (Guillois, Ledoux \& Reynaud 1999).

One the other hand, diamond aggregates have ${ }^{12} \mathrm{C} /{ }^{13} \mathrm{C}$ of $92-93$ (e.g., Russell, Arden \& Pillinger 1996), close to the solar ratio of 89 . The ${ }^{14} \mathrm{~N} /{ }^{15} \mathrm{~N}$ ratio of $417-419$ (Lewis et al. 1987; Russell, Arden \& Pillinger 1996) is similar to 435 measured in Jupiter (Owen et al. 2001). This may suggest that only a portion of pre-solar diamonds formed in supernovae and prompted the quest for the diamonds' other stellar sources.

\section{Pre-solar grains from novae}

A handful of pre-solar SiC and graphite grains have low ${ }^{12} \mathrm{C} /{ }^{13} \mathrm{C}$, low ${ }^{14} \mathrm{~N} /{ }^{15} \mathrm{~N}$, and high ${ }^{26} \mathrm{Al} /{ }^{27} \mathrm{Al}$ ratios (Amari et al. 2001), consistent with theoretical predictions for ejecta of both CO and ONe novae (Starrfield, Gehrz \& Truran 1997; 1998; José \& Hernanz 1998; José et al. 2004). All grains have ${ }^{30} \mathrm{Si}$ excesses $\left({ }^{30} \mathrm{Si} /{ }^{28} \mathrm{Si}\right.$ up to $2.1 \times$ solar $)$. The peak temperatures reached in $\mathrm{CO}$ novae are not high enough to significantly modify $\mathrm{Si}$ isotopic compositions, hence the $\mathrm{Si}$ isotopes of the grains indicate they formed in ONe novae. However, to reproduce the grain data it is necessary to mix pure nova ejecta with a huge amount (99\%) of close-to-solar material (Amari et al. 2001). The apparent lack of known graphite and $\mathrm{SiC}$ grains from $\mathrm{CO}$ novae agrees with expectations from condensation calculations for nova ejecta (José et al. 2004).

Nittler \& Hoppe (2005) argued that grains with low ${ }^{12} \mathrm{C} /{ }^{13} \mathrm{C}$ and ${ }^{14} \mathrm{~N} /{ }^{15} \mathrm{~N}$ ratios could also form in supernovae. Isotopic analysis of $\mathrm{Ti}$ in the grains could distinguish the two stellar sources because Ti isotopes are not modified in novae. Nittler et al. (2006) found a $\mathrm{SiC}$ grain with ${ }^{12} \mathrm{C} /{ }^{13} \mathrm{C}=1$, which can only be obtained by hot $\mathrm{H}$ burning, pointing clearly toward a nova origin.

\section{Conclusions}

Pre-solar grains from SNe and novae exist. Laboratory analysis of such grains gives us, with unprecedented precision, detailed information about the nucleosynthesis products and grain formation from these stars.

\section{Acknowledgements}

This work is supported by NASA grants NNG04GG13G (SA and KL), NNG05GF81G (SA).

\section{References}

Amari, S., Anders, E., Virag, A. \& Zinner, E. 1990, Nature, 345, 238

Amari, S., Gao, X., Nittler, L. R., et al. 2001, ApJ, 551, 1065

Amari, S., Hoppe, P., Zinner, E., \& Lewis, R. S. 1992, ApJ (Letters), 394, L43

Amari, S., \& Zinner, E. 1997, in: T. J. Bernatowicz \& E. Zinner (eds.), Astrophysical Implications of the Laboratory Study of Pre-solar Materials, (New York: AIP), p. 287

Amari, S., Zinner, E., \& Lewis, R. S. 1995, ApJ (Letters), 447, L147 
Bernatowicz, T., Fraundorf, G., Ming, T., et al. 1987, Nature, 330, 728

Bernatowicz, T. J., Amari, S., \& Lewis, R. S. 1992, Lunar Planet. Sci., XXIII, 91

Bernatowicz, T. J., Amari, S., Zinner, E. K., \& Lewis, R. S. 1991, ApJ (Letters), 373, L73

Choi, B.-G., Huss, G. R., Wasserburg, G. J., \& Gallino, R. 1998, Science, 282, 1284

Clayton, D. D., \& Nittler, L. R. 2004, ARAA, 42, 39

Guillois, O., Ledoux, G., \& Reynaud, C. 1999, ApJ (Letters), 521, L133

Hoppe, P., Strebel, R., Eberhardt, P., Amari, S., \& Lewis, R. S. 1996, Science, 272, 1314

Hoppe, P., Strebel, R., Eberhardt, P., Amari, S., \& Lewis, R. S. 2000, Meteorit. Planet. Sci., 35,1157

Hutcheon, I. D., Huss, G. R., Fahey, A. J., \& Wasserburg, G. J. 1994, ApJ (Letters), 425, L97

José, J., \& Hernanz, M. 1998, ApJ, 494, 680

José, J., Hernanz, M., Amari, S., Lodders, K., \& Zinner, E. 2004, ApJ, 612, 414

Lewis, R. S., Tang, M., Wacker, J. F., Anders, E., \& Steel, E. 1987, Nature, 326, 160

Lodders, K., \& Amari, S. 2005, Chem. Erde, 65, 93

Maas, R., Loss, R. D., Rosman, K. J. R., et al. 2001, Meteorit. Planet. Sci., 36, 849

Meikle, W. P. S., Allen, D. A., Spyromilio, J., \& Varani, G.-F. 1989, MNRAS, 238, 193

Messenger, S., Keller, L. P., \& Lauretta, D. S. 2005, Science, 309, 737

Messenger, S., Keller, L. P., Stadermann, F. J., Walker, R. M., \& Zinner, E. 2003, Science, 300, 105

Meyer, B. S., Clayton, D. D., \& The, L.-S. 2000, ApJ (Letters), 540, L49

Nagashima, K., Krot, A. N., \& Yurimoto, H. 2004, Nature, 428, 921

Nguyen, A. N., \& Zinner, E. 2004, Science, 303, 1496

Nittler, L. R., Alexander, C. M. O'. D., Gao, X., Walker, R. M., \& Zinner, E. K. 1994, Nature, 370,443

Nittler, L. R., Alexander, C. M. O'D., \& Nguyen, A. N. 2006, Meteorit. Planet. Sci., 41, A134

Nittler, L. R., Alexander, C. M. O'D., Wang, J., \& Gao, X. 1998, Nature, 393, 222

Nittler, L. R., Amari, S., Zinner, E., Woosley, S. E., \& Lewis, R. S. 1996, ApJ (Letters), 462, L31

Nittler, L. R., \& Hoppe, P. 2005, ApJ (Letters), 631, L89

Nittler, L. R., Hoppe, P., Alexander, C. M. O'D., et al. 1995, ApJ (Letters), 453, L25

Owen, T., Mahaffy, P. R., Niemann, H. B., Atreya, S., \& Wong, M. 2001, ApJ (Letters), 553, L77

Pellin, M. J., Davis, A. M., Calaway, W. F., et al. 2000, Lunar Planet. Sci. XXXI, Abstract \#1934

Pellin, M. J., Davis, A. M., Lewis, R. S., Amari, S., \& Clayton, R. N. 1999, Lunar Planet. Sci. XXX, Abstract \#1969

Pellin, M. J., Davis, A. M., Savina, M. R., Kashiv, Y., Clayton, R. N., Lewis, R. S., \& Amari, S. 2001, Lunar Planet. Sci. XXXII, Abstract \#2125

Richter, S., Ott, U., \& Begemann, F. 1998, Nature, 391, 261

Russell, S. S., Arden, J. W., \& Pillinger, C. T. 1996, Meteorit. Planet. Sci., 31, 343

Starrfield, S., Gehrz, R. D., \& Truran, J. W. 1997, in: T. J. Bernatowicz \& E. Zinner (eds.), Astrophysical Implications of the Laboratory Study of Pre-solar Materials (New York: AIP), 203

Starrfield, S., Truran, J. W., Wiescher, M. C., \& Sparks, W. M. 1998, MNRAS, 296, 502

Tang, M., \& Anders, E. 1988, Geochim. Cosmochim. Acta, 52, 1235

Travaglio, C., Gallino, R., Amari, S., Zinner, E., Woosley, S., \& Lewis, R. S. 1999, ApJ, 510, 325

Travaglio, C., Gallino, R., Zinner, E., Amari, S., \& Woosley, S. 1998, in: N. Prantzos \& S. Harissopulos (eds.), Nuclei in the Cosmos V (Paris: Editions Frontires), p. 567

Woosley, S. E., \& Weaver, T. A. 1995, ApJS, 101, 181

Zinner, E. 1998, Ann. Rev. Earth Planet. Sci., 26, 147 